\title{
Estimation of absolute GNSS satellite antenna group delay variations based on those of absolute receiver antenna group delays
}

\author{
Susanne Beer ${ }^{1}$ (1) $\cdot$ Lambert Wanninger $^{1} \cdot$ Anja Heßelbarth $^{1}$
}

Received: 9 October 2020 / Accepted: 19 April 2021 / Published online: 9 June 2021

(c) The Author(s) 2021

\begin{abstract}
GNSS satellite and receiving antennas exhibit group delay variations (GDV), which affect code pseudorange measurements. Like antenna phase center variations, which affect phase measurements, they are frequency-dependent and vary with the direction of the transmitted and received signal. GNSS code observations contain the combined contributions of satellite and receiver antennas. If absolute GDV are available for the receiver antennas, absolute satellite GDV can be determined. In 2019, an extensive set of absolute receiver antenna GDV was published and, thus, it became feasible to estimate absolute satellite antenna GDV based on terrestrial observations. We used the absolute GDV of four selected receiver antenna types and observation data of globally distributed reference stations that employ these antenna types to determine absolute GDV for the GPS, GLONASS, Galileo, BeiDou, and QZSS satellite antennas. Besides BeiDou-2 satellites whose GDV are known to reach up to $1.5 \mathrm{~m}$ peak-to-peak, the GPS satellites show the largest GDV at frequencies L1 and L5 with up to 0.3 and $0.4 \mathrm{~m}$ peak-to-peak, respectively. They also show the largest satellite-to-satellite variations within a constellation. The GDV of GLONASS-M satellites reach up to $25 \mathrm{~cm}$ at frequency G1; Galileo satellites exhibit the largest GDV at frequency E6 with up to $20 \mathrm{~cm}$; BeiDou-3 satellites show the largest GDV of around $15 \mathrm{~cm}$ at frequencies B1-2 and B3. Frequencies L2 of GPS IIIA, E1 of Galileo FOC, and B2a/B2b of BeiDou-3 satellites are the least affected. Their variations are below $10 \mathrm{~cm}$.
\end{abstract}

Keywords Absolute group delay variations · Code-minus-carrier combination · GPS · GLONASS · Galileo · BeiDou · QZSS

\section{Introduction}

Most of the precise positioning applications of global navigation satellite systems (GNSS) are based on phase observations and, among other things, on the knowledge of the exact positions of the antenna phase centers (APC) of the transmitting and receiving antenna for all frequencies used. Therefore, geodetic-grade receiver antennas are calibrated to determine APC corrections, which can be separated into a mean phase center offset (PCO) and direction-dependent phase center variations (PCV; Rothacher et al. 1995; Wübbena et al. 2000; Zeimetz and Kuhlmann 2008). Based on terrestrial GNSS observations and absolute receiver APC corrections, the GNSS satellite APC can also be determined as it is done by the International GNSS Service (IGS 2019;

Susanne Beer

susanne.beer@tu-dresden.de

1 Geodätisches Institut, Technische Universität Dresden, 01062 Dresden, Germany
Johnston et al. 2017; Schmid et al. 2016). As a result, APC corrections of receiver and satellite antennas can be used independently from each other and in any combinations.

Concerning the GNSS code observables, the counterparts to APC corrections are group delay variations (GDV). GDV depend on antenna type and signal frequency. They vary with nadir angle and elevation angle of the transmitted and received signal, respectively. If not corrected, they degrade the quality of precise applications of the code observable. Only a few absolute GDV calibrations of receiver antennas were published in the past (Wübbena et al. 2008; Kersten and Schön 2017; Breva et al. 2019). Whenever satellite antenna GDV were estimated, observations of one or a set of terrestrial or space-borne receiver antennas provided the reference and, thus, the contributions of satellite and receiver antenna GDV could not be separated properly. Only recently, in the preparation of the third reprocessing campaign (Repro3) for IGS's contribution to the latest International Terrestrial Reference Frame (ITRF; Rebischung 2019; IGS 2019; Villiger et al. 2020), the German company 
Geo++ published multi-GNSS and multi-frequency APC corrections for a subset of receiver antenna types which are used in Repro3 (Wübbena et al. 2019). In this context, corresponding GDV were published, too. Based on these absolute receiver antenna GDV we are now able to estimate absolute multi-frequency GNSS satellite antenna GDV for GPS, GLONASS, Galileo, BeiDou, and QZSS. Since no such receiver antenna GDV corrections were available in earlier studies (Wanninger et al. 2017; Beer et al. 2020), the results for satellite antenna GDV differ from earlier estimations, especially at frequencies with large receiver antenna GDV.

We first summarize previous work on GDV. Then we take a close look at the absolute GDV patterns of four selected receiver antenna types that are the basis for this study. Another section describes our method to estimate satellite antenna GDV based on terrestrial observations of reference stations equipped with these four receiver antennas. It includes subsections dedicated to the data sets used, the required corrections, and a validation of the absolute receiver antenna GDV. The results for the different GNSS are presented and discussed.

\section{Previous work on GDV}

Previous work on GDV is based on different methods and serves various purposes. In the context of precise orbit determination (POD), receiver and satellite antenna GDV were estimated by analyzing either terrestrial or spaceborne observation residuals. Haines et al. (2015) determined nadir-dependent GDV for the entire GPS constellation of 2002-2004. They reach up to $1 \mathrm{~m}$ for the ionospheric-free linear combination (IF) of the frequencies L1 and L2 and show block-specific differences and satellite-to-satellite variations in Blocks IIR-A and IIR-B/M. These nadir-dependent GDV were used as corrections for the realization of a terrestrial reference frame with GPS. Zehentner (2016) determined frequency-specific GDV for the entire GPS constellation and corrected raw observations of low earth orbiting (LEO) satellites to estimate precise orbits for the observation of the earth's time-variable gravity field. His patterns are also azimuth-dependent, but the GDV show mainly nadirdependent variations that reach up to around $20 \mathrm{~cm}$ for L1 and $10 \mathrm{~cm}$ for $\mathrm{L} 2$.

Kovach and Powell (2010) and Hauschild et al. (2012b) analyzed the exceptionally large GDV of GPS space vehicle number (SVN) 49 that stem from satellite-internal multipath and reach up to a few meters. They used pseudorange residuals and the so-called code-minus-carrier combination (CMC), also known as linear multipath combination, respectively.
The CMC approach was also used by several groups to study the large GDV of the BeiDou-2 satellites (Hauschild et al. 2012a; Wanninger and Beer 2015; Yang et al. 2016; Guo et al. 2016; Zou et al. 2017). For frequency B1-2, they amount to $1.5 \mathrm{~m}$ in the case of the medium earth orbit (MEO) satellites and $0.8 \mathrm{~m}$ in the case of the inclined geosynchronous orbit (IGSO) satellites. The more recent BeiDou-3 satellites are not affected by large GDV. They are comparable to those of GPS Block IIR-M or IIF (Zhou et al. 2018).

Wanninger et al. (2017) analyzed the CMC of terrestrial observations and obtained frequency-specific relative satellite and receiver antenna GDV for GPS. Their results are consistent with those mentioned above on the level of $10 \mathrm{~cm}$ root mean square (RMS) for the IF. It was shown that their correction significantly improves single-frequency precise point positioning (PPP), ambiguity-fixing with the Hatch-Melbourne-Wübbena linear combination, and the determination of ionospheric total electron content.

The CMC approach was also applied to observations of reference stations to estimate Galileo and GLONASS GDV (Beer et al. 2020). Homogenous results for antennas of the same satellite type indicate mainly receiver antenna specific GDV. Maximum values reach up to $35 \mathrm{~cm}$ and $30 \mathrm{~cm}$ peakto-peak for Galileo and GLONASS frequencies E1 and G1, respectively.

In principle, absolute receiver antenna GDV can be calibrated with the same methods as used for the calibration of absolute APC, but as far as we know, only two groups estimated absolute receiver antenna GDV based on robot calibrations thus far. Wübbena et al. (2008) published elevation-dependent receiver antenna GDV for six geodetic-grade antennas for GPS frequencies L1 and L2, which reach up to approximately $0.5 \mathrm{~m}$ peak-to-peak in the case of L1. The group at Leibniz University Hannover, Germany, estimated absolute GDV mainly for non-geodetic-grade antennas (Kersten and Schön 2017). The three geodetic-grade antennas they tested show variations of $30-40 \mathrm{~cm}$, but no significant azimuth-dependencies. Now, with the published receiver antenna calibrations in Wübbena et al. (2019), we aim to get clearer insights into the frequency-specific satellite antenna GDV of the different GNSS.

\section{Absolute receiver antenna GDV}

The German company Geo++, which calibrates receiver antennas with their robot device (Wübbena et al. 1997, 2000), presented multi-GNSS and multi-frequency GDV corrections for 36 receiver antenna types. They comprise ten GPS, GLONASS, Galileo, and BeiDou frequencies. Since no digital data sets were made available, we had to digitize the GDV corrections from Wübbena et al. (2019). 
We are using GDV corrections of four receiver antenna types that are widely used in global reference station networks, namely JAVRINGANT_DMINONE, TRM59500.00INONE, TRM59800.00ISCIS, and LEIAR25.R4ILEIT, see Fig. 1. Table 1 lists the GNSS frequency bands according to the nominal frequencies. The digitized GDV corrections exhibit a frequency dependency. In general, the smallest variations occur for the highest frequencies. The least affected frequencies with variations of only a few centimeters are L6/ E6 of the two TRM59800.00 and the LEIAR25.R4 antennas. For all four antenna types, frequency L5/E5a/B2a is the most affected one with variations of almost $30 \mathrm{~cm}$ for TRM59800.00INONE.

The receiver antenna GDV are absolute, i.e., independent from any reference antenna, and type means of individual antenna calibrations. As stated in Wübbena et al. (2019), the published GDV are corrections and they are related to a nominal PCO. We assume that it is the PCO of GPS frequency L1. Thus, the published values show the remaining GDV after applying the L1 PCO to the code observables of all frequencies. On the one hand, that means that PCO differences between L1 and other frequencies are included in the variations. On the other hand, the L1 PCO has to be applied to the code observations of all frequencies to obtain the overall correction.

\section{Method}

Our estimation of absolute multi-frequency and multi-GNSS satellite antenna GDV is based on terrestrial observations of globally distributed reference stations and the code-minuscarrier (CMC) linear combination, which is also known as the linear multipath combination. For detailed descriptions of
Table 1 GNSS frequency bands for which absolute receiver antenna GDV have been published or for which we estimate satellite GDV

\begin{tabular}{llllll}
\hline Frequency $[\mathrm{MHz}]$ & GPS & GLONASS & Galileo & BeiDou & QZSS \\
\hline 1176.450 & L5 & & E5a & B2a & L5 \\
1191.795 & & & E5 & B2 & \\
1202.025 & & G3 & & & \\
1207.140 & & & E5b & B2b & \\
1227.600 & L2 & & & & L2 \\
1246.000 & & G2 & & & \\
1268.520 & & & & B3 & \\
1278.750 & & & E6 & & L6 \\
1561.098 & & & & B1-2 & \\
1575.420 & L1 & & E1 & B1 & L1 \\
1602.000 & & G1 & & & \\
\hline
\end{tabular}

GLONASS frequencies G1 and G2 refer to slot number $k=0$

estimating GDV with the CMC approach, please refer to Wanninger and Beer (2015), Wanninger et al. (2017), and Beer et al. (2020).

Since we corrected the observations for the receiver antenna GDV from Wübbena et al. (2019), only the satellite antenna GDV remained in the CMC linear combination. We modeled them as polynomials of degree four, but did not extract GDV offsets, i.e., GDV offsets were fixed to zero.

While GDV of receiver antennas are given as a function of the elevation angle $e$, satellite antenna GDV were estimated as a function of the nadir angle $\eta$, fixed to zero at zero degrees nadir angle. Both angles are related by

$\sin \eta=\frac{R}{A} \cdot \cos e$

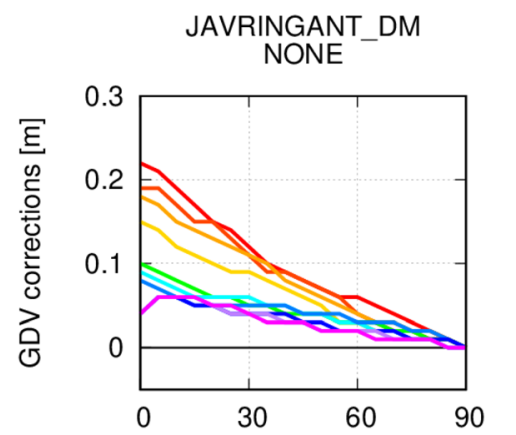

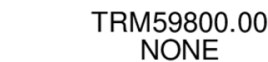

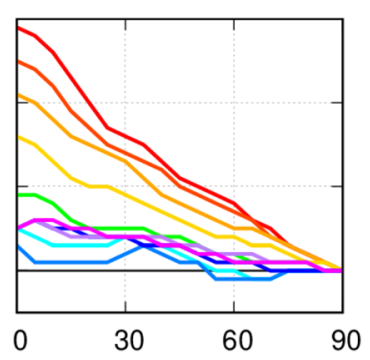

Elevation [deg]
TRM59800.00 SCIS

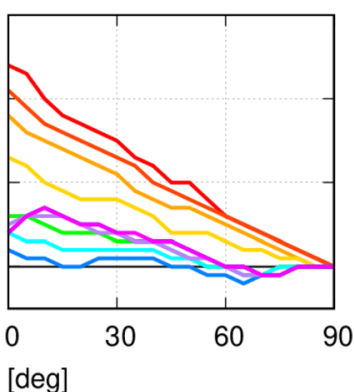

1207.140

1561.098
1227.600

1575.420
LEIAR25.R4 LEIT

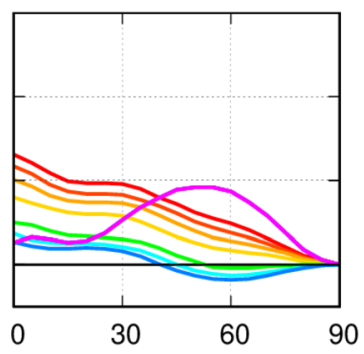

$-1246.000$

1602.000

Fig. 1 Absolute GDV corrections of four types of receiver antennas for ten frequencies, digitized from Wübbena et al. (2019) with a resolution of $5^{\circ}$ in elevation. The GNSS frequency bands are listed in Table 1 according to their nominal frequencies in $\mathrm{MHz}$ 
where $R$ is the earth's radius and $A$ the geocentric distance of the satellite (Rothacher et al. 1995). The term $R / A$ is a constellation-specific constant. Maximum nadir angles for observations of MEO satellites on the earth's surface reach $13.2^{\circ}$ to $14.5^{\circ}$. The maximum nadir angle for IGSO orbits is $8.7^{\circ}$.

We estimated satellite antenna GDV for all frequencies for which absolute receiver antenna GDV were published by Wübbena et al. (2019) if a sufficient number of observation data was available. For GLONASS G3, for which no GDV corrections were published, we applied the GDV corrections of the very similar frequency E5b/B2b.

Since instrumental delays could not be separated from the involved phase ambiguities, the absolute GDV level remains unknown, and only variations could be retrieved. Note that what we refer to as "absolute" in this paper is the independence of the satellite antenna GDV from the receiver antenna GDV.

\section{Data}

As shown in Fig. 2, we selected stations from IGS network, Geoscience Australia, EUREF Permanent GNSS Network, and UNAVCO equipped with one of the four antenna types and which provide observations of as many GNSS and frequencies as possible. However, not all stations provide Galileo E6 or GLONASS G3 observations. BeiDou B1 and B2a observations are available only from stations with the TRM59800.00 antenna, B2b observations of BeiDou-3 MEO satellites are provided only by the stations equipped with a LEIAR25.R4 antenna, and only the Asia-Pacific stations contribute to the GDV estimation of the IGSO satellites.

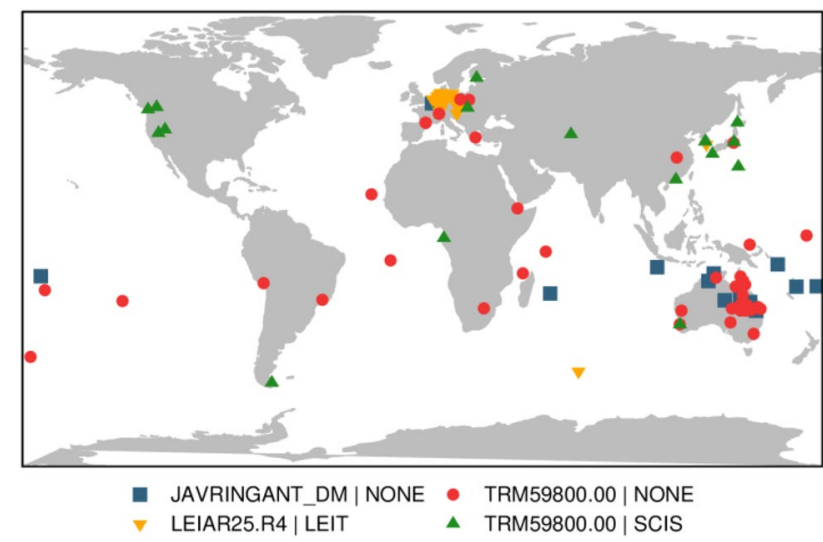

Fig. 2 Thirteen, 41, 17, and 17 reference stations equipped with JAVRINGANT_DMINONE (blue square), TRM59800.00INONE (red circle), TRM59800.00|SCIS (green triangle), and LEIAR25. R4INONE (orange inverted triangle) antennas, respectively, for which absolute receiver antenna GDV are available
For the estimation of nadir-dependent satellite antenna GDV, we needed observations covering the entire elevation range. Because of the daily orbit repeat cycle of GPS, a globally distributed set of reference stations was necessary for this system. For the other MEO constellations, it was important to use data of a whole orbit repeat cycle but there was no need for globally distributed stations (Beer et al. 2020). In the case of the IGSO satellites, reference stations in the Asia-Pacific region were needed. Since temporal stability is assumed of GDV (Beer and Wanninger 2018), we used observations of ten, eight, and seven days for Galileo, GLONASS, and BeiDou MEO satellites, respectively, corresponding to one orbit cycle each. For GPS, QZSS, and BeiDou IGSO satellites, we used three days of observations corresponding to three orbit cycles. All observation data are of February and March 2020.

Observations of a large number of stations should be included to reduce site-specific multipath. Therefore, we accepted mixed tracking modes and mixed receiver models but excluded stations with multipath exceeding $0.5 \mathrm{~m}$ RMS between $10^{\circ}$ and $90^{\circ}$ elevation. Because of the large number of stations and observations, we processed only those arcs spanning an elevation range of at least $60^{\circ}$.

\section{Antenna phase center corrections}

We applied the phase center corrections of ANTEX-file igsR3_2077.atx (IGS 2019). Also, Geo++'s absolute receiver antenna GDV refer to these corrections. Table 2 gives an overview of its content and our assumptions. By applying phase center corrections to the phase observables, our estimated GDV refer to the satellites' center of mass.

The PCO and PCV of GPS and GLONASS satellites are IF values and, thus, identical values are used on the first and second frequency. We applied them also to the third frequency of GPS and GLONASS satellites because no other values are available. These IF PCO values are not the geometric distances of the phase centers at the individual frequencies. However, as long as they do not differ from their real values by more than $0.5 \mathrm{~m}$, their impact on estimated GDV stays below a few centimeters (Wanninger et al. 2017). Concerning GPS, we used the phase center corrections of the first Block IIIA satellite also for the second Block IIIA satellite. For the G3 phase centers of GLONASS-M+ and GLONASS-K1 satellites, we made changes according to Montenbruck et al. (2015).

For Galileo in-orbit validation (IOV) and full operational capability (FOC) satellites, igsR3_2077.atx contains frequency-specific APC corrections (GSA 2020). They are results of ground calibrations and were used for the estimation of new satellite antenna z-offsets for GPS and GLONASS in preparation of IGS's Repro3 (Rebischung 2019). 


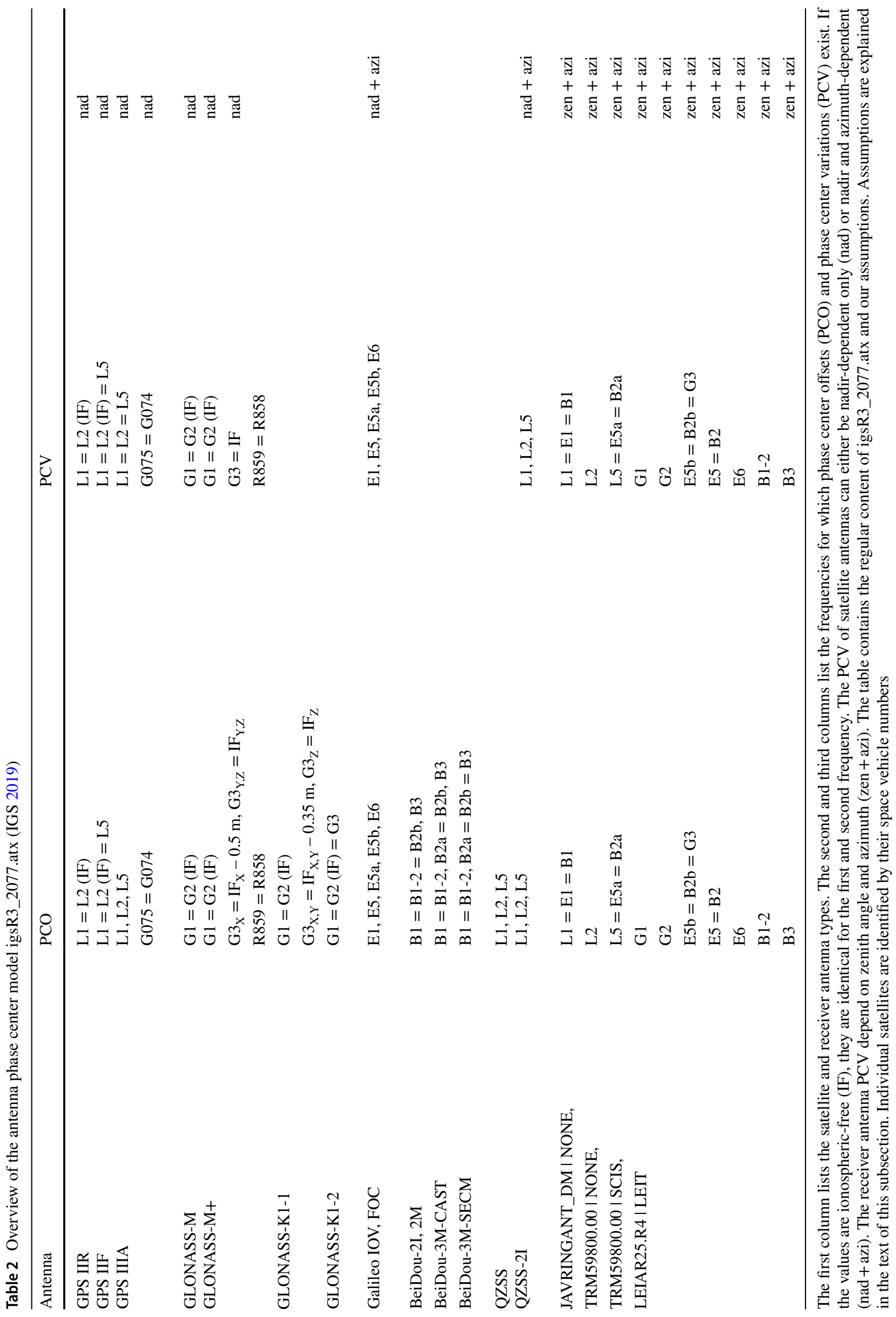


Rec. ant. GDV uncorrected Rec. ant. GDV corrected

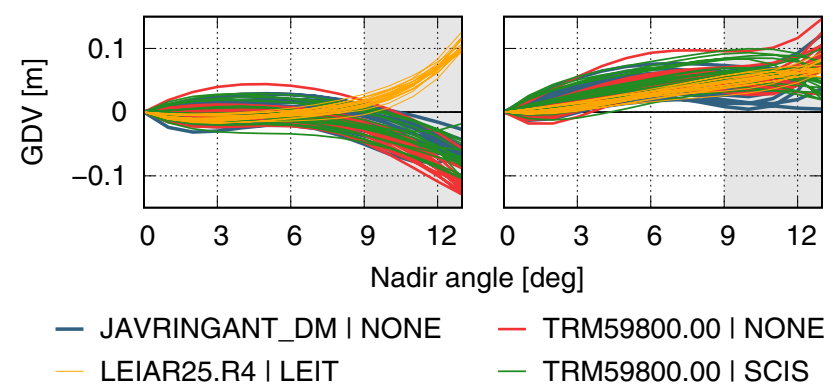

Fig. 3 Receiver antenna type-specific GDV estimations for the Galileo FOC satellite antennas at frequency E5a. Each curve represents the GDV of one satellite; the colors represent the results obtained from the different receiver antennas. The left/right panel is based on uncorrected/corrected receiver antenna GDV, respectively

Table 3 RMS of differences between all possible pairs of four individual satellite GDV estimations based on receiver antenna GDV uncorrected and corrected observations

\begin{tabular}{lllc}
\hline Satellite antenna & Frequency & \multicolumn{2}{l}{$\begin{array}{l}\text { RMS [cm] } \\
\text { Receiver antenna GDV }\end{array}$} \\
\cline { 3 - 4 } & & uncorrected & corrected \\
\hline Galileo FOC & E1 & 5.3 & 4.0 \\
& E5 & 5.2 & 2.3 \\
& E5a & 7.4 & 2.5 \\
& E5b & 5.5 & 2.9 \\
GLONASS-M & E6 & 4.2 & 5.1 \\
& G1 & 8.8 & 11.5 \\
BeiDou-3 M & G2 & 5.0 & 5.1 \\
& B1-2 & 7.3 & 7.0 \\
& B3 & 5.0 & 4.3 \\
\hline
\end{tabular}

The antenna phase center model for Repro3 does not include satellite phase center corrections for BeiDou and QZSS. Therefore, we had to apply the values of the standard IGS correction set igs14.atx (Rebischung et al. 2016a, b) and copied values of neighboring frequencies.

Concerning the receiver antenna types, multi-frequency and multi-GNSS phase center calibrations are stored in igsR3_2077.atx. PCO and PCV are frequency-specific, and the PCV are functions of azimuth and zenith angle. We applied the APC values of E5b/B2b also to frequency G3.

\section{Validation of receiver antenna GDV}

The correctness of our satellite antenna GDV estimation strongly depends on the correctness of the applied receiver antenna GDV and their digitization. For their validation, we used the individual sets of observations of the four types of receiver antennas and estimated each satellite GDV without and with receiver antenna GDV applied. Especially, when the GDV of receiver antennas differ, their correction should produce satellite antenna GDV, which agree much better. Figure 3 shows these GDV estimations for the Galileo FOC satellites at frequency E5a, as an example. Corresponding satellite antenna GDV estimations were conducted for all satellite types comprising at least 15 individual satellites if the number of observations in all four receiver antenna groups was sufficiently large.

Table 3 summarizes the RMS of differences between all possible pairs of the four individual estimations. Since the curves are fit to zero at zero degrees nadir angle, the RMS was calculated for the highest $5^{\circ}$ nadir angle, marked gray in Fig. 3. The frequencies E1, G1, and B1-2, which are close together, show the least improvements by applying the digitized receiver antenna GDV corrections, in the case of G1 even deterioration. However, at most of the other frequencies, a significant improvement is achieved. According to the validation results, we conclude that the receiver antenna GDV are accurate on the level of a few centimeters for most frequencies.

\section{Absolute satellite antenna GDV}

Figure 4 shows the final results of our absolute satellite antenna GDV estimation, where we used the observations of all four receiver antenna types. These GDV are corrections that should be applied with a positive sign to the lefthand side of an observation equation. Table 4 lists the largest peak-to-peak variations.

The results comprise all satellites for which observations were available in the data sets we used. They cover different satellite types within five GNSS. See color-coded curves in Fig. 4. Due to the requirements of the CMC approach, see Sect. "Method", we could not estimate satellite GDV for the Indian Regional Navigation Satellite System (IRNSS) NavIC (only single-frequency observations are available), as well as for geostationary satellites (almost constant elevation at a single receiving station). Concerning BeiDou-3 IGSO satellites and BeiDou-3S satellites, the number of observations was not sufficient.

Most satellite antennas of the same satellite block or generation are affected by similar GDV. The most pronounced satellite-to-satellite differences are exhibited among GPS IIF and IIR satellites at frequencies L5 and L1, respectively, which also show the largest GDV. Pronounced satellite-tosatellite differences among GPS satellites were also found in earlier studies (Haines et al. 2015; Wanninger et al. 2017). As in previous studies, among the GPS IIR-M satellites, SVN G055 shows the largest variations. They amount to 

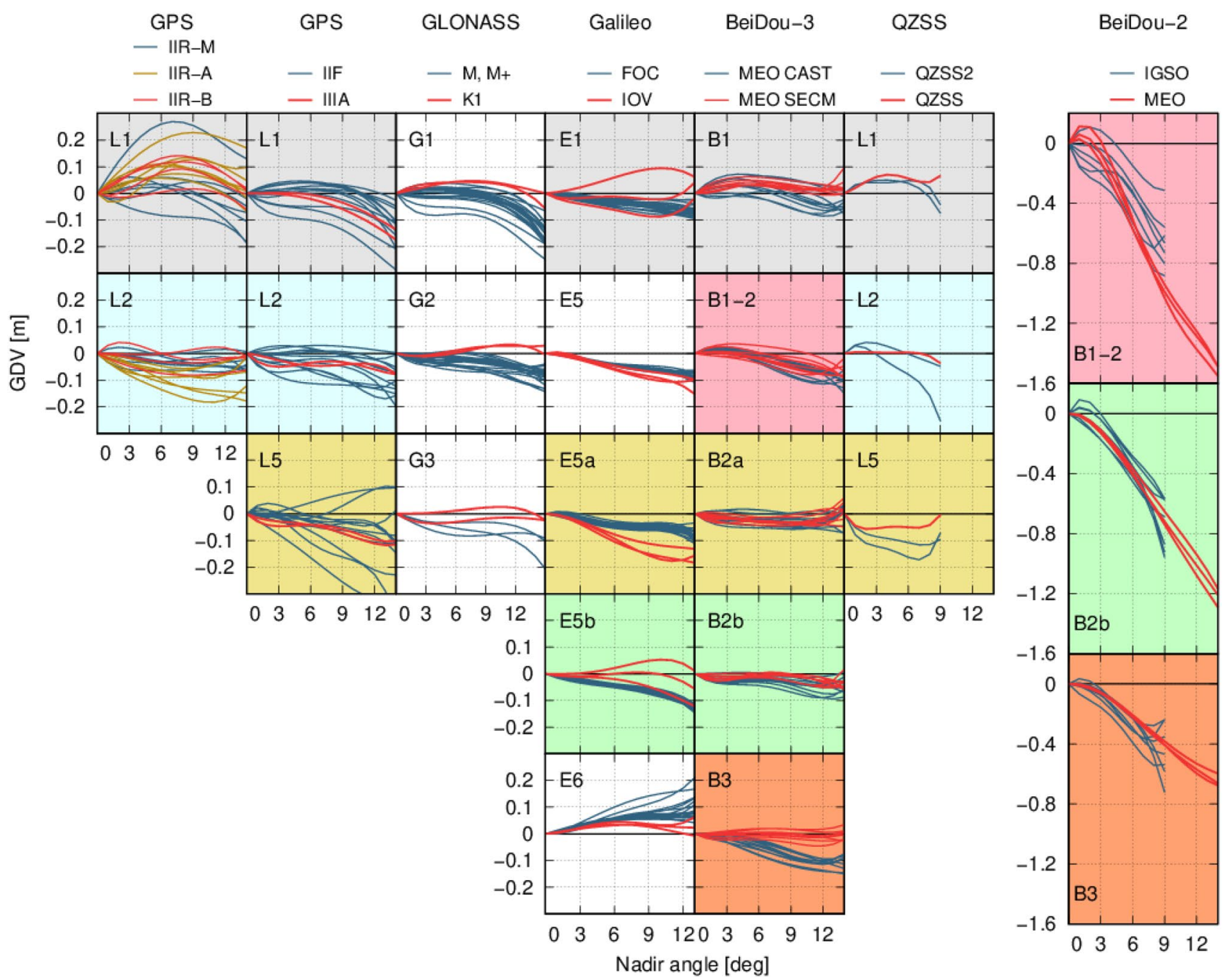

Fig. 4 Absolute GDV corrections for the satellite antennas. Each curve represents the GDV corrections of one satellite. GDV corrections of different satellite types within the same GNSS are drawn in

almost $30 \mathrm{~cm}$ peak-to-peak at frequency L1. GPS IIR-A satellite SVN G041 shows similar GDV. The curves of the two latest Block IIIA satellites fit to the curves of the Block IIF satellites at all frequencies.

In the case of GLONASS and the frequencies G1 and G2, the GDV of the newer GLONASS-K1 satellites fit together well, but differ from the older GLONASS-M and GLONASS-M+ satellites up to $20 \mathrm{~cm}$. At frequency G3, all GLONASS satellites show inhomogeneous GDV curves, especially for high nadir angles. The reason may be found in fewer observations due to a smaller number of satellites transmitting signals at G3.

Except for frequency E6, all GDV of Galileo FOC satellites fit together well. Differences between individual satellites are below $10 \mathrm{~cm}$. The differences between Galileo IOV and FOC satellites and the satellite-to-satellite variations of the IOV satellites might be caused by their lower transmit blue, brown, and red. Colored backgrounds indicate identical frequencies. Due to much larger variations, the BeiDou-2 GDV corrections are printed separately in a different scale

power and lower carrier-to-noise density ratio (Zaminpardaz and Teunissen 2017).

The GDV curves of individual BeiDou-3 MEO CAST and SECM satellites are in good agreement at all frequencies. The GDV at frequencies B1 and B2a have similar orders of magnitude as the Galileo GDV at identical frequencies E1 and E5a. Compared to the exceptionally large satellite antenna GDV of the BeiDou-2 satellites with peak-to-peak differences of up to $1.5 \mathrm{~m}$, the introduced receiver antenna GDV of around $20 \mathrm{~cm}$ are small. Thus, the results for the BeiDou-2 satellite antenna GDV are very similar to those of earlier studies where no receiver antenna GDV were introduced.

GDV effects may increase in linear combinations as demonstrated for the IF in Fig. 5. The satellite antenna GDV of Galileo FOC satellites amount to approximately $0.1 \mathrm{~m}$ when forming the IF of frequencies E1 and E5a. 
Table 4 Maximum variations (peak-to-peak values) of the satellite antenna GDV

\begin{tabular}{|c|c|c|c|}
\hline \multirow{2}{*}{$\begin{array}{l}\text { GNSS/frequency } \\
\text { GPS }\end{array}$} & \multicolumn{3}{|c|}{ Satellite type/maximum variation $[\mathrm{cm}]$} \\
\hline & IIR & IIF & IIIA \\
\hline L1 & 27 & 29 & 18 \\
\hline $\mathrm{L} 2$ & 18 & 20 & 8 \\
\hline L5 & & 41 & 12 \\
\hline GLONASS & M & $\mathrm{M}+$ & $\mathrm{K} 1$ \\
\hline G1 & 25 & 19 & 10 \\
\hline $\mathrm{G} 2$ & 14 & 10 & 4 \\
\hline G3 & & 21 & 5 \\
\hline Galileo & IOV & FOC & \\
\hline E1 & 9 & 9 & \\
\hline E5 & 16 & 11 & \\
\hline E5a & 18 & 11 & \\
\hline $\mathrm{E} 5 \mathrm{~b}$ & 12 & 15 & \\
\hline E6 & 6 & 21 & \\
\hline BeiDou-2 & IGSO & MEO & \\
\hline B1-2 & 88 & 166 & \\
\hline $\mathrm{B} 2 \mathrm{~b}$ & 96 & 129 & \\
\hline B3 & 72 & 68 & \\
\hline BeiDou-3 & MEO CAST & MEO SECM & \\
\hline B1 & 11 & 10 & \\
\hline B1-2 & 16 & 11 & \\
\hline $\mathrm{B} 2 \mathrm{a}$ & 9 & 8 & \\
\hline $\mathrm{B} 2 \mathrm{~b}$ & 9 & 6 & \\
\hline B3 & 15 & 5 & \\
\hline QZSS & QZSS & QZSS2 & \\
\hline $\mathrm{L} 1$ & 7 & 12 & \\
\hline $\mathrm{L} 2$ & 4 & 28 & \\
\hline L5 & 6 & 17 & \\
\hline
\end{tabular}

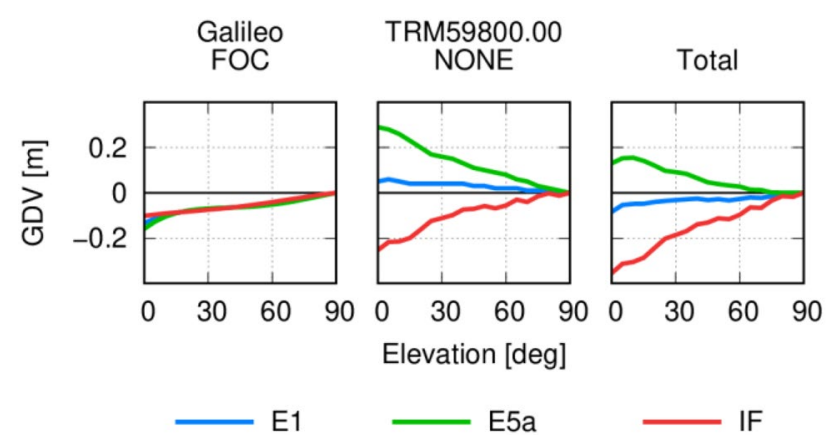

Fig. 5 GDV corrections of the Galileo FOC satellites, one receiver antenna type, and the sum of both at two frequencies and their ionospheric-free linear combination (IF). The satellite antenna GDV were transformed from nadir angle to elevation angle dependence assuming a receiving station on the earth's surface
Together with the GDV of the TRM59800.00INONE receiver antenna, they reach $0.35 \mathrm{~m}$ in total. A similar effect is seen for the IF of frequencies L1 and L5 for most GPS IIF satellites.

\section{Summary}

Recently, an extensive correction set of absolute receiver antenna group delay variations (GDV) was published (Wübbena et al. 2019). By applying these corrections to observations of four types of receiver antennas, we were able to estimate a set of absolute satellite antenna GDV for five GNSS with the code-minus-carrier (CMC) approach. It is the first time that absolute receiver antenna GDV are taken into account in such an estimation process.

The four sets of receiver antenna GDV corrections show a clear frequency dependency. The frequency L5/E5a/B2a at $1176.45 \mathrm{MHz}$ used by GPS, QZSS, Galileo, and BeiDou exhibits the largest variations of up to $30 \mathrm{~cm}$. The frequencies Galileo E6 and GLONASS G1 show the smallest variations of only a few centimeters. No accuracy level was given for the receiver antenna GDV. We validated them by estimating the same satellite antenna GDV four times based on observations separated into the four types of receiver antennas. The results agree on the level of a few centimeters root mean square (RMS) at most frequencies.

The GNSS satellite antennas exhibit GDV that range from very few centimeters up to $1.5 \mathrm{~m}$. The frequencies $\mathrm{B} 2 \mathrm{a} / \mathrm{B} 2 \mathrm{~b}$, E1, L2, and G2/G3 of BeiDou-3 MEO, Galileo FOC, GPS IIIA, and GLONASS-K1 satellites, respectively, are the least affected. Besides the exceptionally large GDV of the BeiDou-2 satellites, frequencies GPS L1 and L5 are the most affected. GDV amount to approximately $0.3 \mathrm{~m}$ on these frequencies and show the largest satellite-to-satellite variations.

Acknowledgments A part of this research has been supported by the German Research Foundation (DFG) under grant WA 868/8-1. All observation data used in this study were made available free of charge by the International GNSS Service (IGS), Geoscience Australia, EUREF Permanent Network (EPN), and UNAVCO. The authors are grateful to these institutions and the station operators for their valuable services.

Funding Open Access funding enabled and organized by Projekt DEAL.

Availability of data and materials The absolute GDV corrections of the four receiver antenna types and the satellite antennas of five GNSS are available in a modified ANTEX format from the corresponding author on request.

Open Access This article is licensed under a Creative Commons Attribution 4.0 International License, which permits use, sharing, 
adaptation, distribution and reproduction in any medium or format, as long as you give appropriate credit to the original author(s) and the source, provide a link to the Creative Commons licence, and indicate if changes were made. The images or other third party material in this article are included in the article's Creative Commons licence, unless indicated otherwise in a credit line to the material. If material is not included in the article's Creative Commons licence and your intended use is not permitted by statutory regulation or exceeds the permitted use, you will need to obtain permission directly from the copyright holder. To view a copy of this licence, visit http://creativecommons. org/licenses/by/4.0/.

\section{References}

Beer S, Wanninger L (2018) Temporal stability of GPS transmitter group delay variations. Sensors 18(6):1744. https://doi.org/10. $3390 / \mathrm{s} 18061744$

Beer S, Wanninger L, Heßelbarth A (2020) Galileo and GLONASS group delay variations. GPS Solut 24(1):23. https://doi.org/10. 1007/s10291-019-0939-7

Breva Y, Kröger J, Kersten T, Schön S (2019) Estimation and validation of receiver antenna codephase variations for multi GNSS. 7th international colloquium on scientific and fundamental aspects of GNSS. Zürich, Switzerland. ftp://gssc.esa.int/esa/colloquium/ 2019-Proceedings_Galileo-colloquium.zip

GSA (2020) Galileo satellite metadata. https://www.gsc-europa.eu/ support-to-developers/galileo-satellite-metadata

Guo F, Li X, Liu W (2016) Mitigating BeiDou satellite-induced code bias: taking into account the stochastic model of corrections. Sensors 16(6):909. https://doi.org/10.3390/s16060909

Haines BJ, Bar-Sever YE, Bertiger WI, Desai SD, Harvey N, Sibois AE, Weiss JP (2015) Realizing a terrestrial reference frame using the Global Positioning System. J Geophys Res Solid Earth 120(8):5911-5939. https://doi.org/10.1002/2015JB012225

Hauschild A, Montenbruck O, Sleewaegen J-M, Huisman L, Teunissen PJG (2012a) Characterization of compass M-1 signals. GPS Solut 16(1):117-126. https://doi.org/10.1007/s10291-011-0210-3

Hauschild A, Montenbruck O, Thoelert S, Erker S, Meurer M, Ashjaee J (2012b) A multi-technique approach for characterizing the SVN49 signal anomaly, part 1: receiver tracking and IQ constellation. GPS Solut 16(1):19-28. https://doi.org/10.1007/ s10291-011-0203-2

IGS (2019) Conventions and modelling for Repro3. http://acc.igs.org/ repro3/repro3.html

Johnston G, Riddell A, Hausler G (2017) The International GNSS Service. In: Teunissen PJG, Montenbruck O (eds) Springer handbook of global navigation satellite systems, 1st edn. Springer, Cham, pp 967-982. https://doi.org/10.1007/978-3-319-42928-1

Kersten T, Schön S (2017) GPS code phase variations (CPV) for GNSS receiver antennas and their effect on geodetic parameters and ambiguity resolution. J Geod(6) 91:579-596. https://doi.org/10. 1007/s00190-016-0984-8

Kovach K, Powell T (2010) The interesting Navstar satellite SVN-49. In: Proceedings of the 2010 international technical meeting of the institute of navigation, San Diego, CA, January 2010, pp 952-962

Montenbruck O, Schmid R, Mercier F, Steigenberger P, Noll C, Fatkulin R, Kogure S, Ganeshan AS (2015) GNSS satellite geometry and attitude models. Adv Space Res 56(6):1015-1029. https://doi. org/10.1016/j.asr.2015.06.019

Rebischung P (2019) IGS reference frame working group technical report 2019. In: Villiger A, Dach R (eds) International GNSS service technical report 2019, pp 237-246
Rebischung P, Altamimi Z, Ray J, Garayt B (2016a) The IGS contribution to ITRF2014. J Geod 90(7):611-630. https://doi.org/10.1007/ s00190-016-0897-6

Rebischung P, Garayt B, Altamimi Z (2016b) IGS reference frame working group technical report 2016. In: Villiger A, Dach R (eds) International GNSS service technical report 2016, pp 171-178

Rothacher M, Schaer S, Mervart L, Beutler G (1995) Determination of antenna phase center variations using GPS observations. In: Proceedings of the 1995 IGS workshop, Potsdam, Germany, pp 205-220

Schmid R, Dach R, Collilieux X, Jäggi A, Schmitz M, Dilssner F (2016) Absolute IGS antenna phase center model igs08.atx: status and potential improvements. J Geod 90(4):343-364. https://doi. org/10.1007/s00190-015-0876-3

Villiger A, Dach R, Schaer S, Prange L, Zimmermann F, Kuhlmann H, Wübbena G, Schmitz M, Beutler G, Jäggi A (2020) GNSS scale determination using calibrated receiver and Galileo satellite antenna patterns. J Geod 94(9):93. https://doi.org/10.1007/ s00190-020-01417-0

Wanninger L, Beer S (2015) BeiDou satellite-induced code pseudorange variations: diagnosis and therapy. GPS Solut 19(4):639-648. https://doi.org/10.1007/s10291-014-0423-3

Wanninger L, Sumaya H, Beer S (2017) Group delay variations of GPS transmitting and receiving antennas. J Geod 91(9):1099-1116. https://doi.org/10.1007/s00190-017-1012-3

Wübbena G, Schmitz M, Menge F, Seeber G, Völksen C (1997) A new approach for field calibration of absolute GPS antenna phase center variations. Navigation 44(2):247-255. https://doi.org/10. 1002/j.2161-4296.1997.tb02346.x

Wübbena G, Schmitz M, Menge F, Böder V, Seeber G (2000) Automated absolute field calibration of GPS antennas in real-time. In: Proceedings of ION GPS 2000. Institute of navigation, Salt Lake City, UT, pp 2512-2522

Wübbena G, Schmitz M, Propp M (2008) Antenna group delay calibration with the Geo++ robot. Poster presentation. IGS Analysis workshop. Miami Beach, FL. http://www.geopp.com/pdf/igs08_ gppgdcalibration_f.pdf

Wübbena G, Schmitz M, Warneke A (2019) Geo++ absolute multifrequency GNSS antenna calibration. EUREF AC workshop, Warsaw, Poland. http://www.geopp.com/pdf/gpp_cal125_euref 19_p.pdf

Yang W, Tong H, Pan L, Xu D, Guo W, Yang J (2016) Analysis and correction of BDS code multipath bias. In: China satellite navigation conference (CSNC) 2016 Proceedings, vol III. Springer, Singapore, pp 503-513

Zaminpardaz S, Teunissen P (2017) Analysis of Galileo IOV + FOC signals and E5 RTK performance. GPS Solut 21(4):1855-1870

Zehentner N (2016) Kinematic orbit positioning applying the raw observation approach to observe time variable gravity. Dissertation, Graz University of Technology

Zeimetz P, Kuhlmann H (2008) On the accuracy of absolute GNSS antenna calibration and the conception of a new anechoic chamber. In: Proceedings of the FIG working week. Stockholm, Sweden, pp 14-19

Zhou R, Hu Z, Zhao Q, Li P, Wang W, He C, Cai C, Pan Z (2018) Elevation-dependent pseudorange variation characteristics analysis for the new-generation BeiDou satellite navigation system. GPS Solut 22(3):60. https://doi.org/10.1007/s10291-018-0726-x

Zou X, Li Z, Li M, Tang W, Deng C, Chen L, Wang C, Shi C (2017) Modeling BDS pseudorange variations and models assessment. GPS Solut 21(4):1661-1668. https://doi.org/10.1007/ s10291-017-0645-2 
Publisher's Note Springer Nature remains neutral with regard to jurisdictional claims in published maps and institutional affiliations.

Susanne Beer is a member of the GNSS research group at the Geodetic Institute of the Technische Universität Dresden (TU Dresden). She received her Dipl.-Ing. degree in geodesy from TU Dresden in 2011. Her research focuses on group delay variations (GDV).
Lambert Wanninger is a professor of geodesy at TU Dresden. He has been involved in research on precise GNSS positioning since 1990 . He holds a Dr.-Ing. degree in geodesy from the University of Hannover, Germany, and a habilitation degree in geodesy from TU Dresden.

Anja Heßelbarth is a research associate at TU Dresden. She has been involved in research on precise GNSS positioning since 2005 and holds a Dr.-Ing. degree in geodesy from TU Dresden. 